\title{
Inhomogeneous Connotations across Square, Stoichiometrically-Based Matrices
}

\author{
Matthew S. Fox ${ }^{*}$ \\ Department Of Chemistry, Cheyenne Mountain High School, Colorado Springs, Colorado, United States \\ *Corresponding author: matthew.2.fox@ucdenver.edu
}

Received June 23, 2015; Revised July 05, 2015; Accepted July 14, 2015

\begin{abstract}
In this report we analyze a subset of chemical equations that have equal numbers of elements and unknown coefficients; linear algebraically, these relate to $n \mathrm{X} n$ matrix systems. Here we associate inhomogeneous eigenvector occurrences to structural properties of chemical equations.
\end{abstract}

Keywords: linear algebra, stoichiometry, eigenvectors, eigenvalues

Cite This Article: Matthew S. Fox, "Inhomogeneous Connotations across Square, Stoichiometrically-Based Matrices." American Journal of Applied Mathematics and Statistics, vol. 3, no. 4 (2015): 142-145. doi: 10.12691/ajams-3-4-1.

\section{Introduction}

Linear algebra and chemistry mainly unite in the field of stoichiometry. Here linear algebra has proven to be effective in establishing systematic representations across all types of chemical equations (e.g. combustion, redox, acid/base, etc.) $[1,2,3,4]$. In this report, such representations are used specifically for what we call square chemical equations - or reactions that have the same number of compounds as unknown coefficients in their representative equations. The interest in this subset of chemical equations arises from the chemistry-based matrix systems formerly acknowledged. For any square chemical equation, we find a matrix system of the order $n$ $\mathrm{X} n$ is always established (hence "square"). In [1] I conclude that a proof for why chemical equations can be balanced with linear algebra exists, but never state it. We begin with an extended, explanatory proof.

Consider the characteristic equation

$$
\mathbf{A} \vec{x}_{\lambda}=\lambda \vec{x}_{\lambda} \mid \vec{x}_{\lambda} \neq \overrightarrow{0}
$$

where $\mathbf{A}$ is a square $n \mathrm{X} n$ matrix and $\vec{x}_{\lambda}$ is an eigenvector for one of $n$ unique eigenvalues $\lambda$ [5]. In [1] I describe how a chemical equation $\mathbf{A}_{c}$, for example, where

$$
\begin{aligned}
& \mathbf{A}_{\mathbf{c}}= x_{1} A_{a} B_{b} \ldots N_{n}+x_{2} A_{a} B_{b} \ldots N_{n} \\
&+\cdots+x_{r} A_{a} B_{b} \ldots N_{n} \\
& \rightarrow x_{r+1} A_{a} B_{b} \ldots N_{n}+x_{r+2} A_{a} B_{b} \ldots N_{n} \\
& \quad+\cdots+x_{p} A_{a} B_{b} \ldots N_{n}
\end{aligned}
$$

(where $x_{1}$ through $x_{r}$ denote both the term and unknown coefficient for the compounds reacting, and $x_{r+1}$ through $x_{p}$ denote likewise for those being produced) can be represented by the matrix setting

$$
\mathbf{A} \vec{x}=\left[\begin{array}{cccc}
a_{11} & a_{12} & \cdots & a_{1 p} \\
b_{21} & b_{22} & \cdots & b_{2 p} \\
\vdots & \vdots & \ddots & \vdots \\
n_{p 1} & n_{p 2} & \cdots & n_{p p}
\end{array}\right]\left[\begin{array}{c}
x_{1} \\
x_{2} \\
\vdots \\
x_{p}
\end{array}\right]=\left[\begin{array}{c}
0 \\
0 \\
\vdots \\
0
\end{array}\right],
$$

from simple conservation of mass- that is,

$$
\sum \text { reactants }-\sum \text { products }=0,
$$

where the above equation refers to the same element [6]. Eq 2, however, reformulates to $\mathbf{A} \vec{x}=\overrightarrow{0}$ and, if $\mathbf{A}$ is $n \mathrm{X} n$, then $\lambda=0$ in eq 1 . Thus, if a chemical equation possesses a square coefficient matrix, then there exists at least one $\lambda$ $=0$.

Definition. $n \mathrm{X} n$ coefficient matrices (those derived from square chemical equations), we call chemical matrices.

In [1] I continue to explain how the kernel of a chemical matrix (a consequence of its reduced row echelon form) can yield the solution to the chemical equation. This is of no surprise, however, as we have determined that all chemical equations are linearhomogeneous systems; and therefore have a unique null space (and thus chemical solution) equal to the vector $\vec{X}$ [6].

Such a solution, however, is only one of $n$ for $\mathbf{A}_{c}$, as an $n \mathrm{X} n$ matrix will have $n$ eigenvalues and thus $n$ eigenvectors associated with it. It is therefore the purpose of this work to examine these other linear algebraic significances.

\subsection{Creation of Chemical Matrices}

A majority of chemical equations retain dimensions of $m \mathrm{X} n$ where $m \neq n$. Below we present two methods-the first for the case of $m>n$ and the second for $m<n-$ on creating chemical matrices that can be analyzed with eq 1 . 
Chemically, if $m>n$ then the equation in question has more elements reacting than terms in the equation. For example, the reaction

$$
\mathbf{B}_{\mathrm{C}}=x_{1} \mathrm{AgNO}_{3}+x_{2} \mathrm{~K}_{3} \mathrm{PO}_{4} \rightarrow x_{3} \mathrm{Ag}_{3} \mathrm{PO}_{4}+x_{4} \mathrm{KNO}_{3}
$$

has a total of 5 unique elements, but only 4 terms. We find

$$
\mathbf{B}=\left[\begin{array}{cccc}
1 & 0 & -3 & 0 \\
1 & 0 & 0 & -1 \\
3 & 4 & -4 & -3 \\
0 & 3 & 0 & -1 \\
0 & 1 & -1 & 0
\end{array}\right],
$$

where $\operatorname{dim}(\mathbf{B})=5 \mathrm{X}$ 4. Similar to adding zero to an algebra problem, we add the term $\mathrm{Ag}_{0} \mathrm{~N}_{0} \mathrm{O}_{0} \mathrm{~K}_{0} \mathrm{P}_{0}$ to the product side, yielding the term and coefficient designation $x_{5}$. ${ }^{1}$ Such an addition does not alter the chemical meaning - only the dimensions_of a given chemical equation. We then find

$$
\mathbf{B}^{\prime}=\left[\begin{array}{ccccc}
1 & 0 & -3 & 0 & 0 \\
1 & 0 & 0 & -1 & 0 \\
3 & 4 & -4 & -3 & 0 \\
0 & 3 & 0 & -1 & 0 \\
0 & 1 & -1 & 0 & 0
\end{array}\right],
$$

where $\operatorname{dim}\left(\mathbf{B}^{\prime}\right)=5$ X 5 .

We employ a similar tactic for the case of $m<n$; however, here we append an additional element $\Phi_{0}$ to each term in the non-square equation. ${ }^{2}$ For example, consider the reaction

$$
\mathbf{C}_{\mathrm{c}}=x_{1} \mathrm{H}_{2}+x_{2} \mathrm{O}_{2} \rightarrow x_{3} \mathrm{H}_{2} \mathrm{O} .
$$

Here

$$
\mathbf{C}=\left[\begin{array}{lll}
2 & 0 & -2 \\
0 & 2 & -1
\end{array}\right],
$$

where $\operatorname{dim}(\mathbf{C})=2 \times 3$. Appending $\Phi_{0}$ to each term established the reaction

$$
\mathbf{C}_{\mathrm{C}}^{\prime}=x_{1} \mathrm{H}_{2} \Phi_{0}+x_{2} \mathrm{O}_{2} \Phi_{0} \rightarrow x_{3} \mathrm{H}_{2} \mathrm{O} \Phi_{0},
$$

where

$$
\mathbf{C}^{\prime}=\left[\begin{array}{ccc}
2 & 0 & -2 \\
0 & 2 & -1 \\
0 & 0 & 0
\end{array}\right] .3
$$

Again, this method does not modify a chemical equations' delineation—only its dimensions.

\subsection{Properties of Chemical Matrices}

Lemma 1. For any $n \mathrm{X} n$ chemical matrix $\mathbf{A}, n \geq 2$.

Proof. By definition chemical matrices denote the changes compounds undergo in a reaction. If there is only a single element in the entire reaction $(n=1)$, then this

\footnotetext{
${ }^{1}$ Albeit this term could be appended to either side of the equation, it is simplest to append to the rightmost side of the equation (the $n$th column).

${ }^{2} \Phi_{0}$ could denote any element in the Periodic Table of the Elements; however, due to the zero subscript, it is non-existent in the equation.

${ }^{3}$ Similar to the case of $m>n$, it is easiest to append the $\Phi_{0}$ row vector to the bottommost row of a chemical matrix.
}

element cannot react if the $n \mathrm{X} n$ matrix order is to be maintained. If $n=2$, however, as in the reaction $x_{1} A B \rightarrow x_{2} A_{2} B_{2}$, a reaction does occur, and is therefore the minimum order of a chemical matrix.

Lemma 2. For any $n \mathrm{X} n$ chemical matrix $\mathbf{A}, \operatorname{det}(\mathbf{A})=0$. Proof. By the invertible matrix theorem in [7],

$$
\operatorname{det}(\mathbf{A})=\prod_{k=1}^{n} \lambda_{k}
$$

Because $\exists \lambda=0$, $\operatorname{det}(\mathbf{A})=0$.

Consequently, [7] asserts that the following two conditions are true:

1. the linear transformation $\vec{X} \mapsto \mathbf{A} \vec{x}$ is not one-to-one

2. the columns of $\mathbf{A}$ do not form a linearly independent set.

Albeit other assertions can be made, these are the few that have chemical significance. We will proceed in respective order.

The linear transformation $\vec{x} \mapsto \mathbf{A} \vec{x}$ for chemical matrix A resembles one of the most fundamental laws governing balanced reactions: the law of multiple proportions - that is, "When an element combines with another to form more than one compound the masses of the second element combining with a fixed mass of the first element bear a simple ratio to one another" [8]. For example, the reaction $2 \mathrm{KClO}_{3} \rightarrow 2 \mathrm{KCl}+3 \mathrm{O}_{2}$ is the same as $4 \mathrm{KClO}_{3} \rightarrow 4 \mathrm{KCl}+$ $6 \mathrm{O}_{2}$, which is the same as $2 n \mathrm{KClO}_{3} \rightarrow 2 n \mathrm{KCl}+3 n \mathrm{O}_{2}$, where $n \in \mathbb{R}$. Modeling this reaction linear algebraically produces the system

$$
\left[\begin{array}{ccc}
1 & -1 & 0 \\
1 & -1 & 0 \\
3 & 0 & -2
\end{array}\right] \vec{x}_{0}=\overrightarrow{0}
$$

where both $\vec{x}_{0}$ and $\overrightarrow{0} \in \mathbb{R}^{n}$, and $\vec{x}_{0}$ is the eigenvector for $\lambda=0$. Because it is the ratio of the elements that must be identical, $\vec{x}_{0}$ can be any scalar multiple of itself; therefore, the liner transformation $\vec{x} \mapsto \mathbf{A} \vec{x}$ cannot be one-to-one.

Following eq 3 , the total mass of a system is conserved for any closed reaction. This implies that, as in the previous example, all reactant columns (positive entries) are dependent on all product columns (negative entries), as the sum of unique scalar multiples of these columns must equal the zero-vector (eq 2). Accordingly, the columns of $\mathbf{A}$ do not form a linearly independent set.

Definition. The fact that the commutative property of addition holds for chemical equations, provided the reactants remain on the reactant side and products on the product side, we call chemical commutativity.

Chemical commutativity establishes the idea that chemical matrices with $n>2$ will possess $\geq 2 \lambda=0$ and thus $\geq 2$ chemical solutions. We prove this below.

Let $r$ denote the number of reacting terms in $\mathbf{A}_{\mathrm{c}}$ and $p$ the number being produced.

Lemma 3. For any chemical equation $\mathbf{A}_{c}$, there exists exactly ( $r$ !)(p!) empirical chemical solutions.

Proof. The number of unique arrangements for the reactant side $\left(U_{r}\right)$ in $\mathbf{A}_{\mathrm{c}}$ is given by $U_{r}=r$ !. Similarly, for the product side $U_{p}=p$ !. Consequently, the total number of unique arrangements is $\Pi(U)=(r !)(p !)$. 
This result implies that $(r !)(p !)$ chemically correct matrices exist, where each has at least one $\lambda=0$ by eq 1 .

As trivial as chemical commutativity may seem, it is the basis to obtaining the first inhomogeneous significance we will explore.

\section{Main Results}

As stated in the introduction, the homogeneous equation $\mathbf{A} \vec{x}=\overrightarrow{0}$ is unique to chemical equations as it demonstrates the law of conservation of mass. $\lambda=0$, however, is one of $n$ eigenvalues for a chemical matrix. In this section, we examine occurrences of $\lambda \neq 0$ and the eigenvectors that correspond.

The following assertions will be based on an $n \mathrm{X} n$ chemical matrix $\mathrm{D}$, where

$$
\mathbf{D}=\left[\begin{array}{cccc}
a_{11} & a_{12} & \cdots & a_{1 p} \\
b_{21} & b_{22} & \cdots & b_{2 p} \\
\vdots & \vdots & \ddots & \vdots \\
n_{p 1} & n_{p 2} & \cdots & \mu
\end{array}\right] .
$$

We denote the set of all matrix elements in D that lie upon the main diagonal-that is: $\left\{a_{11}, b_{22}, c_{33}\right.$, etc. $\}$-as $\Gamma_{\mathrm{D}}$.

Definition. Terms in a chemical equation that consist of only one element in the entire substance, we call singular molecules. For example, $\mathrm{O}_{2}$ functions as a singular molecule in the reaction $x_{1} \mathrm{CH}_{4}+x_{2} \mathrm{O}_{2} \rightarrow x_{3} \mathrm{H}_{2} \mathrm{O}+x_{4} \mathrm{CO}_{2}$.

Lemma 4. For any chemical reaction that either reacts or produces a singular molecule $A_{\mu}$, where $A$ is a singular molecule and $\mu$ is A's corresponding subscript, $\exists \geq 1 \lambda=$ $\mu$, provided $\mu \in \Gamma_{\mathbf{D}}$.

Proof. Because $\mu$ represents a singular molecule in the reaction, all other values in the $n$th column of $\mathrm{D}$ must equal zero $[1,3]$. Thus,

$$
\mathbf{D}=\left[\begin{array}{cccc}
a_{11} & a_{12} & \cdots & 0 \\
b_{21} & b_{22} & \cdots & 0 \\
\vdots & \vdots & \ddots & \vdots \\
n_{p 1} & n_{p 2} & \cdots & \mu
\end{array}\right] .
$$

The characteristic polynomial of $\mathbf{D}, P(\mathbf{D})$, can be factored into the form $\prod(\lambda-\tau)$, where $\tau$ denotes a unique number (one of $n$ ) in the factored form of $P(\mathbf{D}) .{ }^{4}$ By definition $P(\mathbf{D})=0$, and thus each $\tau$ denotes an eigenvalue of D. To prove, we find $\operatorname{det}(\mathbf{D})$. By Laplace Expansion

$$
\operatorname{det}(\mathbf{D})= \pm \mu\left|\begin{array}{cccc}
a_{11} & a_{12} & \cdots & a_{1 p-1} \\
b_{21} & b_{22} & \cdots & b_{2 p-1} \\
\vdots & \vdots & \ddots & \vdots \\
n_{p 1} & n_{p 2} & \cdots & n_{p p-1}
\end{array}\right|
$$

Therefore, $(\lambda \mp \mu) \in \Pi(\lambda-\tau)$, or, similarly, $\exists \geq 1 \lambda=\mu$. Lemma 5. For the specific case of $\mu=n_{p p}$ in $\mathbf{D}, \vec{x}_{\mu}=\hat{n}$, where $\vec{x}_{\mu}$ is the eigenvector for $\lambda=\mu$ and $\hat{n}$ is the nth unit-vector in $\mathbb{R}^{n}$.
Proof. Neglecting the trivial solution of $\vec{x}_{\mu}=\overrightarrow{0}$, if we assume $\vec{x}_{\mu}=\widehat{n}$, then

$$
\lambda \vec{x}_{\mu}=\mu \widehat{n}=\left[\begin{array}{c}
0 \\
0 \\
\vdots \\
0 \\
\mu
\end{array}\right]
$$

We find,

$$
\mathbf{D} \hat{n}=\left[\begin{array}{c}
0+0+\cdots+0+0 \\
0+0+\cdots+0+0 \\
\vdots \\
0+0+\cdots+0+0 \\
0+0+\cdots+0+\mu
\end{array}\right]=\left[\begin{array}{c}
0 \\
0 \\
\vdots \\
0 \\
\mu
\end{array}\right] .
$$

Consequently, $\mathbf{D} \vec{x}_{\mu}=\lambda \vec{x}_{\mu}$.

We generalize this point below.

Theorem 1. Provided $\mu \in \Gamma_{\mathbf{D}}$, then $\vec{x}_{\mu} \in\{\hat{i}, \hat{j}, \cdots, \hat{n}\} \in \mathbb{R}^{n}$.

Proof. We assume $\vec{x}_{\mu} \in\{\hat{i}, \hat{j}, \cdots, \widehat{n}\}$, then

$$
\lambda \vec{x}_{\mu}=\mu \vec{x}_{\mu} \in\left\{\left[\begin{array}{c}
\mu \\
0 \\
\vdots \\
0 \\
0
\end{array}\right],\left[\begin{array}{c}
0 \\
\mu \\
\vdots \\
0 \\
0
\end{array}\right], \cdots,\left[\begin{array}{c}
0 \\
0 \\
\vdots \\
0 \\
\mu
\end{array}\right]\right\} .
$$

We find

$$
\mathbf{D} \hat{i}, \mathbf{D} \hat{j}, \cdots, \mathbf{D} \hat{n}=\left[\begin{array}{c}
\mu \\
0 \\
\vdots \\
0 \\
0
\end{array}\right],\left[\begin{array}{c}
0 \\
\mu \\
\vdots \\
0 \\
0
\end{array}\right], \cdots,\left[\begin{array}{c}
0 \\
0 \\
\vdots \\
0 \\
\mu
\end{array}\right],
$$

respectively. This concludes the proof.

Realize that because of chemical commutativity, obtaining the $\vec{x}_{\mu}$ eigenvector is simply a matter of rearranging a chemical matrix to allow $\mu \in \Gamma_{\mathbf{D}}$ (provided a singular molecule in the reaction exists).

We continue by recalling the mathematical implication of the law of conservation of mass (eq 3). Reformulating eq 3 for each element in $\mathbf{A}_{c}$ yields the linear-homogeneous system

$$
\left\{\begin{array}{c}
\left(\sum_{r=1}^{x_{r}} x_{r} a_{r}\right)-\left(\sum_{p=r+1}^{x_{p}} x_{p} a_{p}\right)=0 \leftarrow A \\
\left(\sum_{r=1}^{x_{r}} x_{r} b_{r}\right)-\left(\sum_{p=r+1}^{x_{p}} x_{p} b_{p}\right)=0 \leftarrow B \\
\vdots \\
\left(\sum_{r=1}^{x_{r}} x_{r} n_{r}\right)-\left(\sum_{p=r+1}^{x_{p}} x_{p} n_{p}\right)=0 \leftarrow N
\end{array}\right.
$$

as shown in [1]. Identically, 


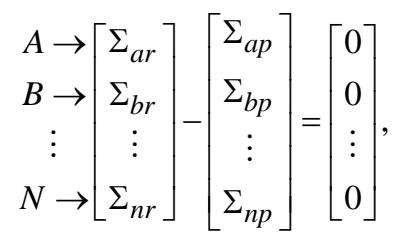

where, for instance, $\Sigma_{a r}=\sum_{r=1}^{x_{r}} x_{r} a_{r}$ for all $A$ elements reacting in $\mathbf{A}_{\mathrm{c}}$ and $\Sigma_{a p}=\sum_{p=r+1}^{x_{p}} x_{p} a_{p}$ for all $A$ elements being produced.

Definition. The vector $\vec{R}$ that results from the substitution of a random coefficient vector $\vec{\zeta}$ into eq 6 , we call residue. ${ }^{5}$

Formally,

$$
\vec{R}=\left[\begin{array}{c}
\Sigma_{a r} \\
\Sigma_{b r} \\
\vdots \\
\Sigma_{n r}
\end{array}\right]-\left[\begin{array}{c}
\Sigma_{a p} \\
\Sigma_{b p} \\
\vdots \\
\Sigma_{n p}
\end{array}\right] \leftarrow A
$$

for any $\vec{\zeta}$ substituted into $\mathbf{A}_{c}$. For example, in the reaction $x_{1} \mathrm{KClO}_{3} \rightarrow x_{2} \mathrm{KCl}+x_{3} \mathrm{O}_{2}$, if we substitute in a random coefficient vector

$$
\vec{\zeta}=\left[\begin{array}{l}
3 \\
2 \\
5
\end{array}\right] \text { for }\left[\begin{array}{l}
x_{1} \\
x_{2} \\
x_{3}
\end{array}\right] \text { respectively, } \vec{R}=\left[\begin{array}{c}
1 \\
1 \\
-1
\end{array}\right] \text {. }
$$

(Obviously, the substitution of $\vec{x}_{0}$ produces $\vec{R}=\overrightarrow{0}$, as in eq 6.) $)^{6}$

Let $\lfloor\vec{A}\rfloor$ denote a vector with entries in their simplest, whole number ratios. We present the following question: are there any values for $\vec{\zeta}$ that satisify $\vec{\zeta}=\lfloor\vec{R}\rfloor$ ? We consider solutions of $\vec{\zeta}=\overrightarrow{0}$ trivial.

Theorem 2. For a chemical matrix A, all $\vec{x}_{\lambda}=\vec{\zeta}$ satisify $\vec{\zeta}=\lfloor\vec{R}\rfloor$.

Proof. Eq 7 is derived from a system of equations similar to eq 5 - that is,

$$
\vec{R}=\left\{\begin{array}{l}
\left(\sum_{r=1}^{\zeta_{r}} \zeta_{r} a_{r}\right)-\left(\sum_{p=r+1}^{\zeta_{p}} \zeta_{p} a_{p}\right)=R_{1} \\
\left(\sum_{r=1}^{\zeta_{r}} \zeta_{r} b_{r}\right)-\left(\sum_{p=r+1}^{\zeta_{p}} \zeta_{p} b_{p}\right)=R_{2}, \\
\vdots \\
\left(\sum_{r=1}^{\zeta_{r}} \zeta_{r} n_{r}\right)-\left(\sum_{p=r+1}^{\zeta_{p}} \zeta_{p} n_{p}\right)=R_{n}
\end{array}\right.
$$

where $R_{n}$ denotes the $n$th element of $\vec{R}$. Equivalently,

\footnotetext{
${ }^{5}$ Note both $\vec{R}$ and $\vec{\zeta} \in \mathbb{C}^{n}$.

${ }^{6}$ The calculation of residue is commonly used to check chemical equations. If $\vec{R}=\overrightarrow{0}, \vec{\zeta}=$ either $\overrightarrow{0}$ or $\vec{x}_{0}$.
}

$$
\vec{R}=\left[\begin{array}{cccc}
a_{11} & a_{12} & \cdots & a_{1 p} \\
b_{21} & b_{22} & \cdots & b_{2 p} \\
\vdots & \vdots & \ddots & \vdots \\
n_{p 1} & n_{p 2} & \cdots & n_{p p}
\end{array}\right]\left[\begin{array}{c}
\zeta_{1} \\
\zeta_{2} \\
\vdots \\
\zeta_{p}
\end{array}\right]=\mathbf{A} \vec{\zeta}
$$

Let $\vec{\zeta}=\vec{x}_{\lambda}$. Then, $\mathbf{A} \vec{x}_{\lambda}=\vec{R}$ with equivalent residue of $\lambda \vec{x}_{\lambda}$ by eq 1 . Therefore, $\quad \mathbf{A} \vec{x}_{\lambda}=\lambda\lfloor\vec{R}\rfloor$, or, indistinguishably, $\vec{x}_{\lambda}=\lfloor\vec{R}\rfloor$.

The chemical connotation associated with $\vec{x}_{\lambda}=\lfloor\vec{R}\rfloor$ asserts that, on the foundations of eqs 6 and 7, other chemical solutions exist linear algebraically. Albeit inhomogeneous, these solutions associate themselves uniquely with different subsets of chemical equations. They are therefore only idiosyncratic to the structure of chemical equations, with no correspondence to chemical reactivity.

\section{Conclusion}

Through the use of linear algebra, specialized subsets of chemical equations were analyzed. In this manuscript, we proved correct the calculator-based balancing method explained in [1]. Introduced was the notion of chemical commutativity, which eventually inaugurated the mathematical implications of singular molecules. We then proved that all chemical equations will have residue $\lambda\lfloor\vec{R}\rfloor$ for any $\vec{x}_{\lambda} \neq \overrightarrow{0}$, which demonstrates that inhomogeneous solutions to chemical equations exist, but only structurally.

\section{Competing Interest}

The author declares no competing financial interest.

\section{Acknowledgements}

This manuscript was supported in part by Janie Mueller, Chuck Hendrick, Dave Hale, Shaun Carmody, Ralph Boedigheimer, and Brad Warner.

\section{References}

[1] Fox, M.S. On Balancing Acidic and Basic Reduction/Oxidation Reactions with a Calculator. World J. of Chem. Educ. 2015, 3 (3), 74-77.

[2] Olson, J.A. An Analysis of the Algebraic Method for Balancing Chemical Reactions. J. Chem. Educ. 1997, 74 (5), 538-542.

[3] Alberty, R.A. Chemical Equations are Actually Matrix Equations. J. Chem. Educ. 1991, 68, 984.

[4] Kennedy, J.H. Balancing Chemical Equations with a Calculator. J. Chem. Educ. 1982, 59, 523.

[5] Strang, G. Linear Algebra and Its Applications, 4th ed.; Academic Press, INC.: London, 2006; pp 73.

[6] McCoy, B. Application of Linear Algebra: Balancing Chemical Equations. The University of North Carolina at Chapel Hill. http://www.unc.edu/\$sim\$marzuola/Math547\_S13/Math547\_ S13\Projects/B\McCoy\Section001\BalancingChemicalEquat ions.pdf (accessed Apr. 2015).

[7] Dawes, J. The Invertible Matrix Theorem-Proofs. The University of Manchester. 2014, 1-4.

[8] Taylor, H.S. The Atomic Concept of Matter. A Treatise on Physical Chemistry. 1942, 1, 2. 